\title{
A clinical computer and monitor
}

\author{
J. R. B. GREER \\ Department of Physiology, University of Glasgow
}

\begin{abstract}
Summary
The key to the early warning of a deteriorating clinical situation lies in the change in parameters rather than in their absolute value. This not only colours the choice of sensor, but also affects the design of the discriminators between the sensors and the logic circuits.

While the sensors and logic are clearly the most important parts of the equipment, it is probably the presentation of information which will in the end decide the extent to which it is used by nursing staff, and every care must be taken to ensure, not only that false alarms are minimized, but that genuine alarms do not cause unnecessary agitation of the patient.
\end{abstract}

EARLIER PAPERS this morning have described many aspects of patient monitoring, but the instrument $I$ should like to discuss arises directly out of $\mathrm{Mr}$ Stewart's concept of monitoring the patient by looking at a number of different parameters and only noting these changes which could have a clinical significance.

This concept was developed in its first form by Mr T. D. Sadera in Professor R. K. Penny's Department of Engineering Design and Production in Liverpool, and preliminary experience with this model indicated that the approach was extremely promising, but that the type of relay logic used raised a number of serious problems, and it was felt that the next move should be to make up a much more sophisticated instrument based on electronic logic, although at this stage no decision was made as to whether this should be discrete or integrated. Each part of the instrument was discussed and planned separately, and it will probably therefore be convenient to start with the problems raised in designing a suitable form of input and proceed through the logic to the display.

\section{Comparators}

It is of course, commonplace that in designing an alarm system of any sort, one has first to decide at at what point the alarm should be set off. In other circumstances this may represent an appropriate density of smoke or of temperature rise in electrical equipment. In this case however, it was quite clear that the signal level that would represent normal conditions for one patient might represent an emergency situation for another patient. Thus it was accepted initially that the selection of appropriate alarm levels would require setting by the clinician in charge of the case, in the light of all the facts in his possession.

Again, when we began to look at the parameters which we hoped to measure, it became clear that problems were going to arise in the selection of suitable sensing devices, since as $\mathrm{Mr}$ Wolff has pointed out, it is often extremely inconvenient-and sometimes downright impossible without insertion of catheters-to monitor continuously those parameters (such as blood pressure) whose numerical values are clinically familiar. This however, is not to say that one may not be able to obtain a signal whose value will give an accurate index of changes in, for example, blood pressure-indeed the more Mr Stewart and I thought about this, the more we felt that there was much to be gained and little to be lost by departing from the requirement to measure absolute values, in a manner that would be numerically compatible with the single measurement procedures from which much clinical data is drawn.

Having reached this decision, the design of the input stage became clear. The clinician would take each channel in turn and adjust the internal reference signal to be equal to the incoming signal from the corresponding sensor. Thus in this case he would have three reference signals representing the initial condition of the patient. Having thus 'tuned' the instrument to the patient, the next move would be to decide, on a percentage basis, what variation from his initial settings should be regarded as significant and possibly dangerous. There would, of course, be no need to set the upper and lower limits in the same way, since anything more than say a $15 \%$ fall in heart rate might be regarded as potentially serious, while a $15 \%$ rise would be well within the expected pattern of recovery. This process of setting the reference signal and later setting the permissible limits was accomplished by three pairs of compara- 


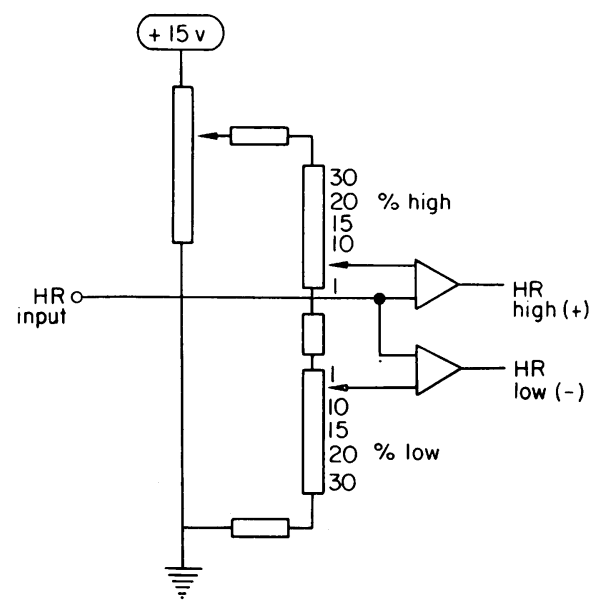

FIG. 1.

tors of which one is shown in Fig. 1, the setting of limits being carried out by suitable alteration to the potentiometer from which each reference voltage was derived (upper panel on Fig. 2). In addition to these high and low comparators, a further three indicated if the input signal fell below a predetermined small figure, this being taken as an indication that the corresponding sensor had become disconnected or had fallen off completely. This completed the first part of the instrument, and we are now in a position that if any sensor produces an output which has changed by more than a given amount from the intial conditions, then a signal is produced inside the instrument, recording an abnormal clinical level. This is displayed (e.g. B.P. HIGH) directly on the front panel of the instrument (lower panel on Fig. 2), and is also fed into the logic system-along with all the other sensor outputs-in order that logic may consider the whole picture and decide whether the change or changes that have occurred add up to make a recognizable clinical picture.

\section{Logic}

The clinical thinking which is the background to the logic system of this instrument has already been fully described by Mr Stewart, and I do not feel that it would be appropriate for me to go into this in any detail. It may, however, be of interest to look briefly at the clinical requirements and how these were turned into electronic circuitry.

To take the case of cardiac arrest, it is clear that this condition would be accompanied by low blood pressure and low heart rate.

In the circuit (Fig. 3) the outputs from the comparators run along the top. Normally there will be no voltage on these lines, but if one or more of the comparators records an abnormal condition, then the appropriate rail will carry a raised voltage level, numbers 1, 3 and 5 indicating abnormally high sensor signals, and numbers 2, 4 and 6 indicating low levels. In the particular case, we may assume an abnormal signal has appeared on lines 2 and 4 (low heart rate and low blood pressure). This information will be brought down by the leads shown as bold lines to the AND gate and hence to the CARDIAC ARREST output,

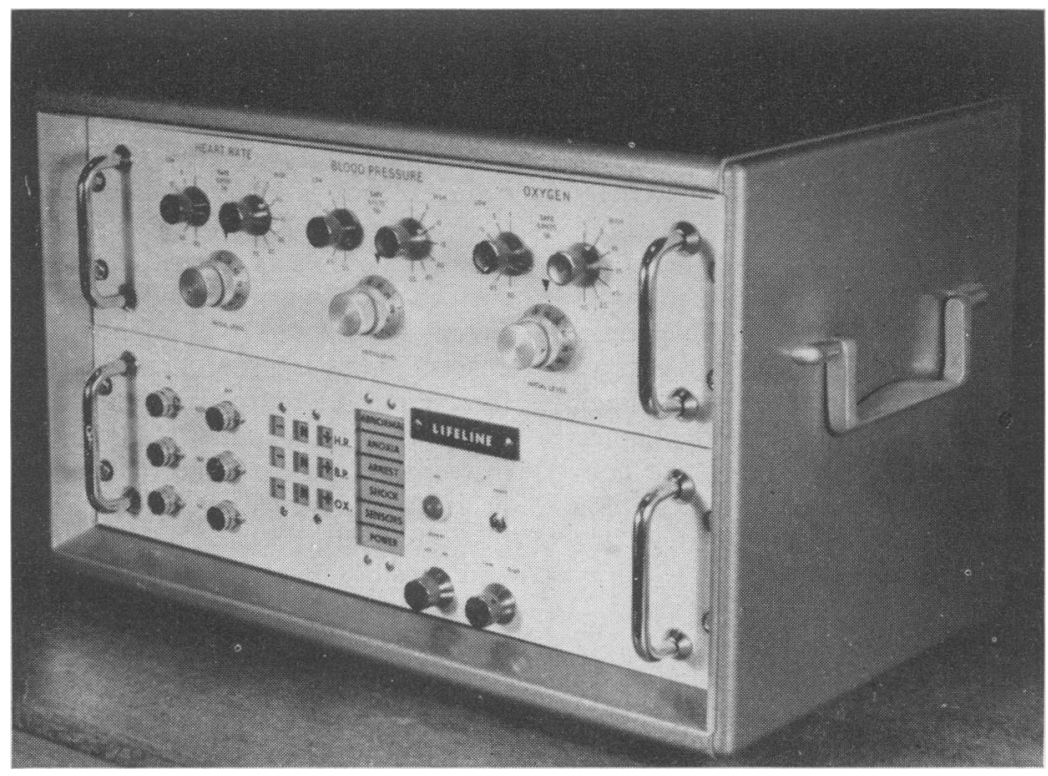

FIG. 2. 


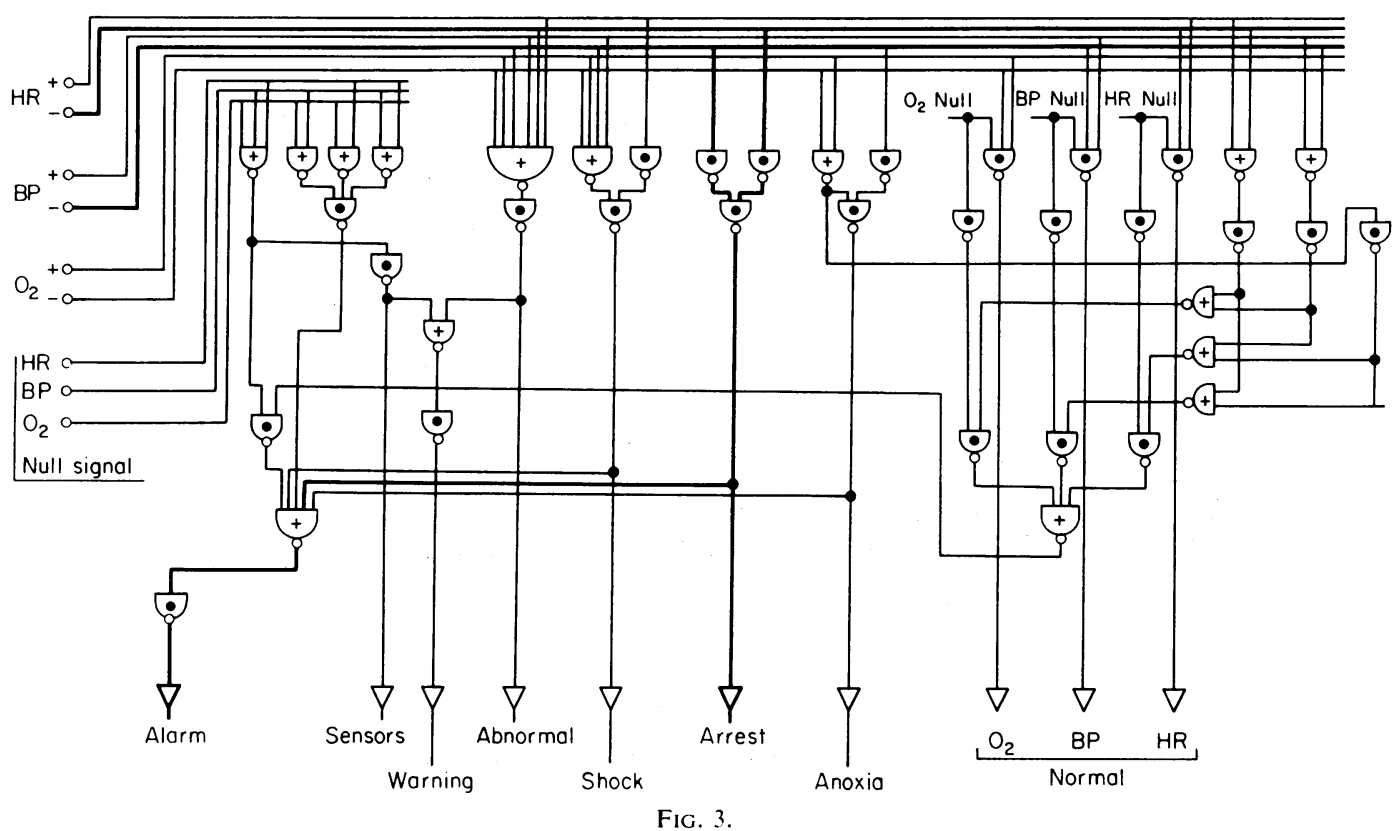

and it will also operate one of the possible inputs to the ALARM channel. It will be seen that the other clinical outputs are similarly derived.

One initial decision which had to be made lay in the choice between integrated circuits and discrete components. In the context of what was after all still an experimental model, the decision did not appear to be completely obvious. There is, of course, no doubt that integrated circuits-which were in fact eventually used-have the great advantage of compactness, but it was realized that there might have been advantages at this stage in using discrete components, in view of the fact that there were relatively few stages in each element of logic and the use of discrete components would have allowed us to retain an actual wiring assembly which closely resembled the logic diagram, and which would among other things have allowed for alteration of the logic if the situation required. Probably in fact, the best answer would have been a compromise between the two, and I think it is likely that the next instrument that is made will use integrated circuits-but will use them if necessary rather extravagantly, in order to ensure that as far as possible each part of the logic which is separate in context, is also separate in construction. Fig. 4 shows the internal assembly of logic boards.

\section{Visual display}

It was clear from the beginning that the form of display was going to be of considerable importance in the usefulness of the finished instrument. It was also agreed that while some form of audible alarm was necessary as a last resort it was hoped that it would prove possible to attract attention, and provide the required information with a minimum of disturbance to the patient. Four types of visual indicators were considered. The most obviously attractive were electroluminescent panels-such as the familiar luminescent panels in aircraft, exhorting one to fasten one's safety-belt-but in addition to these, the mertis of gallium arsenide diodes and tungsten filament bulbs were considered along with meter-type indicators. To take the electroluminescent panels first, these have many advantages. They provide a glowing panel which while clearly legible in all but strong light is never offensive. They do not have a filament to burn out, and they require relatively little power. Initially it had been intended that the entire instrument should be battery operated, and the low dissipation of electroluminescent panels appeared to be another major factor in their favour. For this reason then, they were in fact fitted in preference to the other types of indicator. This, however, was an unwise move and we finally decided to remove the E.L. panel for reasons which are perhaps worth listing. First it should be said that one of their strongest initial attractions had been their economy in current consumption, arising out of their very high efficiency. As we had initially hoped that the equipment could be battery operated, this seemed to be a strong attraction. However, once the indicator assembly was complete, it became clear that the standing drain of the oscillator required to generate the high voltage required for the panels represented 


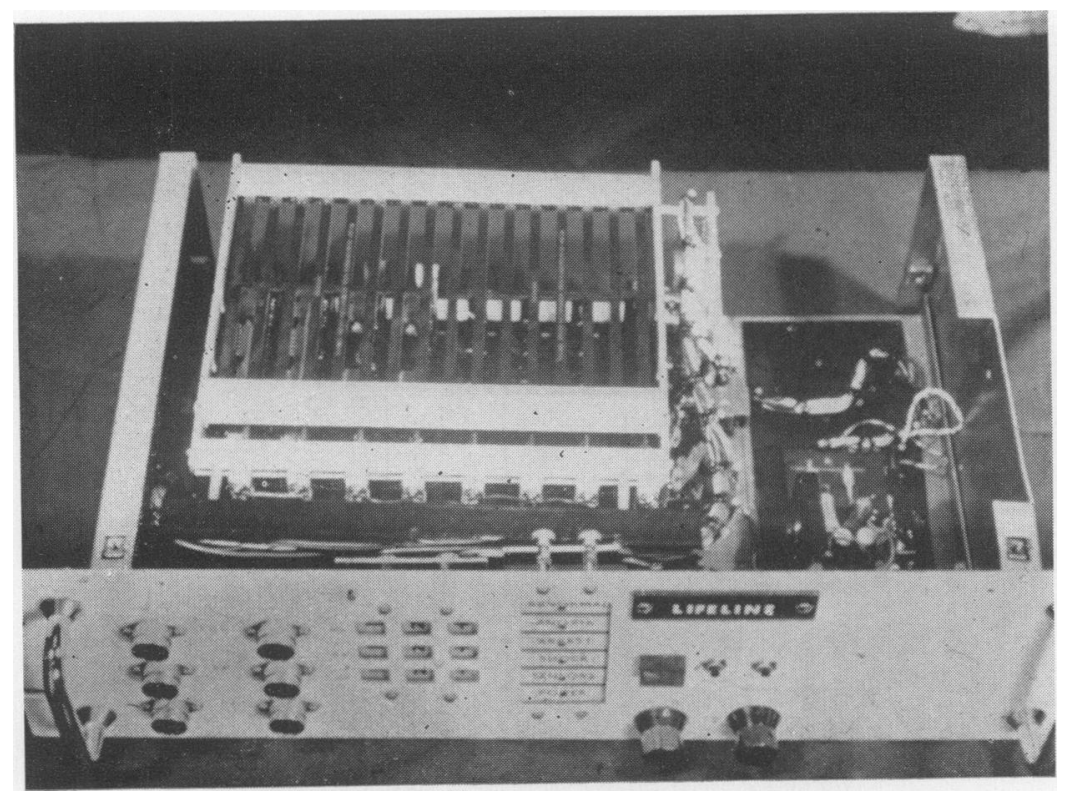

Fig. 4.

a continuing drain almost as large as would have been required by ordinary tungsten bulbs. Moreover, as this oscillator ran at $400 \mathrm{~Hz}$, and its waveform was not particularly smooth, it represented a significant source of interference which caused trouble with the setting of the comparators. The final decision, however, which made us reject these panels resulted from a tendency that they developed (during test) of flickering, even when a steady voltage was applied to them. On closer examination we found that arcing was occurring where the connecting wires were bonded to the ceramic plate. While this had not yet reached the stage of representing a serious failure, we felt that the advantages of reliability were perhaps more apparent than real, in panels as small as this which required a number of separate connections. A second alternative would perhaps have been gallium arsenide diodes and these might well be one of the more attractive forms of low power indicators. On the other hand, they are at best a bright red spot and we therefore felt that it would probably be wise to ensure, at least for an experimental model, that the legends were clearly indicated, and that we were making the best use of colour coding. The tungsten bulbs now in use are under-run and although single bulbs are used in the instrument being demonstrated, they would normally be duplicated, partly in order to provide better panel illumination, and partly on the grounds of reliability. A further type of indicator which we did not explore seriously at this stage but which one would bear in mind for future consideration, is the type of miniature meter which is rather reminiscent of the bell indicator found in Victorian houses. On this type of unit, a miniature drum rotates to change a sector, for example, from green to red. Their obvious advantage would again lie in batterypowered instruments, since the total current required by them would be of the order of a small number of milliamps instead of about 50 milliamps each for quite small tungsten bulbs, and a total drain of 300 milliamps continuously for the oscillator driving the electroluminescent panels.

\section{Audio warnings}

So much then for the different ways of illuminating a visual display. However, it remains to provide some suitable form of audible warning in the event of a visual display alone not attracting attention. As the basis of the audible device, we selected a bleep-tone. This is a sort of miniature loud-speaker with a built in oscillator which provides a clear characteristic tone with a very modest power input. As there are no mechanical parts in the sense of an electric bell, it is also reliable and can easily be turned on or off by transistor switches. It was decided that two levels of warning would be required.

First, a bleeping tone would be generated by a low speed multi-vibrator and this used to provide a quiet indication that one of the sensors has become detached, or some similar non-critical situation had occurred. If, however, the logic in the box decided that one of the three clinical alarm conditions had occurred, then at the same time as the word ANOXIA, CARDIAC ARREST or SHOCK was illuminated on the 
panel, a loud and continuous note would be emitted from the bleep-tone until such time as the instrument was reset. I should perhaps say at this stage that we would not now feel that this was a desirable form of display and instead, when a clinical situation arose, first a warning light would come on, so that if any medical staff were in the vicinity they would look at the patient, cancel the warning on the instrument, and take any necessary steps to deal with the clinical problem, before in fact the patient was aware that anything was regarded as having gone amiss. In the event of the visible signs not being observed, after a short delay very quiet tones would become audible and this would gradually build up over a period of several minutes, the intention of this being, of course, that if the initial visual display was not seen, a quiet tone would probably attract staff in the vicinity, and only in last resort would a loud tone be reached. It should, perhaps, be emphasized at this stage that the equipment is regarded as being of a self-contained nature, and therefore the advantages of generating a warning at some distance from the instrument cannot be assumed.

\section{Power}

Reference has been made from time to time to a shift in view as to the desirable power supplies. It was initially felt that in the interests of safety and also portability, the instrument should be entirely batterypowered. However, as time progressed, it became clear that many of the sensors with which it was likely to be used, either would require to be driven from the mains or else could only be used on batteries for relatively short periods. Even where they were apparently suitable for battery operation a large number of different sizes of battery was going to be required, and therefore we would be faced with the necessity of either changing numerous batteries according to a regular programme, or running all equipment off a small number of batteries, but using stabilizing circuits to generate different voltages-a procedure which is in general uneconomic of battery power. Thus it had become apparent that the advantages of portability that would arise from operating the instrument from batteries would be largely lost when it was connected up to the essential sensors: moreover, from the point of view of safety, there seemed little merit in having the main instrument battery powered, when some at least of the sensors were likely to be mains operated. A very similar situation arose from the point of view of safety. With the removal of the electroluminescent panels, no high voltages are required inside the logic instrument, and indeed since all the relays are sealed reed relays it would seem that with ordinary precautions such as using carefully screened and earthed mains transformers, the patient hazards should be minimal. It was therefore felt that on balance mains operation was likely to offer the most satisfactory service, although the possibility of reverting to battery operation would be very seriously borne in mind once tests have been completed and suitable sensors finally selected. 